\title{
Salvage brachytherapy in combination with interstitial hyperthermia for locally recurrent prostate carcinoma following external beam radiation therapy: a prospective phase II study
}

\author{
Andrzej M. Kukietka, MD, PhD', Prof. Vratislav Strnad, MD, PhD², Prof. Paul Stauffer, MSc, PhD, \\ Tomasz Dąbrowski, MD, PhD4, Marcin Hetnał, MD, PhD', Damian Nahajowski, MSc', Tomasz Walasek, MD, PhD4, \\ Piotr Brandys, MD, $\mathrm{PhD}^{5}$, Robert Matys, $\mathrm{MD}^{6}$ \\ 'Amethyst Radiotherapy Center, Ludwik Rydygier Memorial Hospital, Kraków, Poland, ${ }^{2}$ Department of Radiation Oncology, University \\ Hospital Erlangen, Erlangen, Germany, ${ }^{3}$ Radiation Oncology Department, Thomas Jefferson University, Philadelphia PA, USA, ${ }^{4}$ Department \\ of Radiotherapy, Maria Skłodowska Curie Memorial Centre of Oncology, Kraków Division, Kraków, Poland, ${ }^{5}$ Oncology Clinic, Breast and Chest \\ Unit, Maria Skłodowska Curie Memorial Centre of Oncology, Kraków Division, Kraków, Poland, 'Holy Cross Cancer Center, Kielce, Poland
}

\begin{abstract}
Optimal treatment for patients with only local prostate cancer recurrence after external beam radiation therapy (EBRT) failure remains unclear. Possible curative treatments are radical prostatectomy, cryosurgery, and brachytherapy. Several single institution series proved that high-dose-rate brachytherapy (HDRBT) and pulsed-dose-rate brachytherapy (PDRBT) are reasonable options for this group of patients with acceptable levels of genitourinary and gastrointestinal toxicity. A standard dose prescription and scheme have not been established yet, and the literature presents a wide range of fractionation protocols. Furthermore, hyperthermia has shown the potential to enhance the efficacy of re-irradiation.

Consequently, a prospective trial is urgently needed to attain clear structured prospective data regarding the efficacy of salvage brachytherapy with adjuvant hyperthermia for locally recurrent prostate cancer. The purpose of this report is to introduce a new prospective phase II trial that would meet this need. The primary aim of this prospective phase II study combining Iridium-192 brachytherapy with interstitial hyperthermia (IHT) is to analyze toxicity of the combined treatment; a secondary aim is to define the efficacy (bNED, DFS, OS) of salvage brachytherapy. The dose prescribed to PTV will be 30 Gy in 3 fractions for HDRBT, and 60 Gy in 2 fractions for PDRBT. During IHT, the prostate will be heated to the range of $40-47^{\circ} \mathrm{C}$ for 60 minutes prior to brachytherapy dose delivery. The protocol plans for treatment of 77 patients.
\end{abstract}

J Contemp Brachytherapy 2015; 7, 3: 254-258 DOI: 10.5114/jcb.2015.51871

Key words: clinical trial, high-dose-rate brachytherapy, hyperthermia, prostate cancer, pulsed-dose-rate brachytherapy.

\section{Purpose}

Approximately $10 \%$ of low-risk group patients and up to $60 \%$ of high-risk group patients will experience biochemical recurrence (BF) [1-3]. Generally accepted and most commonly applied definition of biochemical failure after definitive RT is the Phoenix definition (PSA increase of $>2 \mathrm{ng} / \mathrm{ml}$ above the nadir reached after RT) [4]. Metastatic or both metastatic and local disease are found in about $50 \%$ of patients with biochemical progression who have undergone previous radiotherapy. Local recurrence only is found in $25 \%$ of patients with biochemical pro- gression. The majority of local recurrences occur at the site of dominant primary tumour [5].

There is no consensus with respect to optimal treatment in previously irradiated patients with sole local recurrence of prostate adenocarcinoma. There are only two potentially curative treatment modalities for previously irradiated recurrent prostate cancer: salvage surgery or salvage brachytherapy; these have very similar efficacy but different toxicities [6]. Salvage prostatectomy series have demonstrated durable long term control. However, aggressive salvage treatment options should be offered with great care, because reported failure rates as well as
Address for correspondence: Andrzej M. Kukiełka, MD, PhD, Amethyst Radiotherapy Center, Ludwik Rydygier Memorial Hospital, Kraków, Poland, os. Złotej Jesieni 1, Kraków, Poland, phone/fax: +48 1234091 34, e-mail: drkukielka@gmail.com
Received: 30.03 .2015

Accepted: 14.05.2015

Published: 25.06.2015 
toxicity rates can be significant, especially in inexperienced hands, regardless of salvage technique [7]. Other treatment possibilities such as cryotherapy or high-intensity focused ultrasound (HIFU) are useful only for carefully selected patients. In addition, HIFU is currently not recommended as an alternative to accepted curative treatment approaches for localized prostate cancer [8].

Androgen deprivation should be considered a palliative treatment. It is also not well tolerated by most patients and has many side effects, e.g. insulin resistance and diabetes, bone loss, fatigue, sexual dysfunction (erectile impotence, loss of libido), symptomatic gynecomastia, hot flushes, anaemia, and cardiovascular events. Before deciding to perform local salvage therapy, metastases to regional lymph nodes and distant metastases should be ruled out. It is also crucial to identify patients that are most likely to have only local disease. Factors that are associated with better biochemical control included post-treatment PSA nadir of $<0.5 \mathrm{ng} / \mathrm{ml}$, presalvage PSA $<10$, Gleason score $<8$, PSADT (PSA doubling time) $>6$ months, disease free interval $>24$ months, and low risk initial tumor [6].

To date, there remains no consensus on standard dose and fractionation scheme for salvage prostate HDR brachytherapy. Previously published series of cases, as well as ABS guidelines contain a wide range of prescribed doses and various fractionation schemes [9].

Prostate brachytherapy allows the administration of a high radiation dose in a small volume of tissue. Both temporary implants (Ir-192) and permanent implants (I-125, Pd-103) have been used for treatment of prostate cancer recurrence after EBRT. There are few available publications on HDRBT or PDRBT; most of them are retrospective studies that apply to small groups of patients [10-16]. The results and toxicity are summarized in the Table 1.

Preclinical studies have clearly demonstrated that hyperthermia has the potential to enhance radiation re- sponse by a factor of 1.4-2.0 [17]. In patients with radioresistant prostate cancer, the addition of hyperthermia could potentially break down radiation resistance and increase the chance of cure.

Consequently, a prospective trial is urgently needed to attain clear structured prospective data regarding the efficacy of salvage brachytherapy with adjuvant hyperthermia for locally recurrent prostate cancer. The purpose of this report is to introduce a new prospective phase 2 trial that will evaluate the safety and effectiveness of temporary brachytherapy in combination with interstitial hyperthermia as a treatment for local recurrence of prostate cancer after EBRT. The study will examine the side effects of combined treatment, as well as its ability to cure the cancer. A protocol of this clinical trial was designed by the radiation oncologists from Krakow (Poland) and Erlangen (Germany) as an international multicenter study.

\section{Material and methods}

\section{Study design}

This is a prospective, multicenter non-randomized phase II study. Patients are recruited during the radiation therapy consultation in participating centers.

\section{Eligibility criteria}

Men with biopsy-confirmed locally recurrent prostate adenocarcinoma in stage $\mathrm{T} 1 \mathrm{~b}-\mathrm{T} 3 \mathrm{~b} \mathrm{~N} 0 / \mathrm{pN} 0 \mathrm{M0}$ are eligible for the study. Biopsy should be performed less than 6 months before registration and more than 24 months after initial EBRT. As evaluated with a TRUS volume study performed 0-4 weeks before registration, each patient should meet the eligibility criteria for prostate brachytherapy as follows: prostate/tumor volume $<60 \mathrm{ml}$, the distance between rear prostate edge and rectal mucosa $>5 \mathrm{~mm}$, interference of pubic arch ruled out, and, if local stage T3b, it must be possible to cover the entire can-

Table 1. Toxicity and outcomes of salvage brachytherapy for prostate cancer recurrence after external beam irradiation

\begin{tabular}{|c|c|c|c|c|c|}
\hline Study, year & Salvage therapy & $n$ & $\begin{array}{l}\text { Median follow-up } \\
\text { (months) }\end{array}$ & Grade $3 / 4$ toxicity & Outcome \\
\hline Łyczek et al. [10] & $\begin{array}{c}\text { HDR } \\
30 \mathrm{~Gy} / 3 \mathrm{fx} / 3 \mathrm{impl}\end{array}$ & 115 & No information & $\begin{array}{c}12.2 \% \mathrm{GU} \\
0.9 \% \mathrm{Gl}\end{array}$ & $\begin{array}{l}46 \% \text { bPFS in patients with PSA }<6 \\
18 \% \text { bPFS in patients with PSA }>6\end{array}$ \\
\hline Scala et al. [11] & $\begin{array}{c}\text { HDR } \\
36 \mathrm{~Gy} / 6 \mathrm{fx} / 2 \mathrm{impl}\end{array}$ & 10 & 18.5 & $\begin{array}{l}70 \% \text { grade } 2 \\
\text { or greater }\end{array}$ & $70 \%$ dfs \\
\hline Jo et al. [12] & $\begin{array}{c}\text { HDR } \\
22 \mathrm{~Gy} / 2 \mathrm{fx} / 1 \mathrm{impl}\end{array}$ & 11 & 29 & ०\% & $63 \%$ bNED \\
\hline Morikawa et al. [13] & $\begin{array}{c}\text { HDR } \\
32 \mathrm{~Gy} / 4 \mathrm{fx} / 1 \mathrm{impl}\end{array}$ & 29 & 22 & $0 \%$ & $79 \%$ bDFS \\
\hline Chen et al. [14] & $\begin{array}{c}\text { HDR } \\
36 \mathrm{~Gy} / 6 \mathrm{fx} / 2 \mathrm{impl}\end{array}$ & 52 & 59.6 & $2 \%$ & $51 \% 5 y$ bDFS \\
\hline Lahmer et al. [15] & $\begin{array}{c}\text { PDR } \\
60 \mathrm{~Gy} / 2 \mathrm{impl}\end{array}$ & 18 & 21 & $0-17 \%$ & 57\% PSA-rfsp \\
\hline Kukiełka et al. [16] & $\begin{array}{c}\text { HDR + IHT } \\
30 \mathrm{~Gy} / 3 \mathrm{fx} / 3 \mathrm{impl}\end{array}$ & 25 & 13 & $0 \%$ & $74 \% 2 y$ bDFS \\
\hline
\end{tabular}


cer infiltration by the prescribed minimum brachytherapy dose.

The exclusion criteria are: TURP/prostatic salvage cryosurgery/HIFU performed more than 6 months before registration, previous prostatectomy, androgen deprivation therapy within 3 months prior to registration, performance status ECOG $>2$, International Prostate Symptoms Score (IPSS) $>20$, PSA doubling time $<6$ months, baseline gastrointestinal (GI) or genitourinary (GU) toxicity grade $\geq 2$ as defined in Common Terminology Criteria for Adverse Events (CTCAE) version 4.0 [18]. Written study-specific informed consent will be obtained from all the patients before study registration.

\section{Primary endpoint}

To estimate the rate of late Grade $\geq 3$ gastrointestinal (GI) and genitourinary (GU) toxicities related to salvage treatment for locally recurrent prostate cancer after previous external beam radiation therapy. Treatment-related toxicity will be evaluated according to the Common Toxicity Criteria for Adverse Events version 4.03 (CTCAE).

\section{Secondary endpoints}

1. To estimate the rate of acute treatment-related GI/ GU adverse events. 2. To determine time to biochemical failure (Phoenix definition [4])/ biochemical disease-free survival (bDFS), overall survival (OS), disease-free survival (DFS), disease-specific survival (DSS), and clinical patterns of tumor recurrence (time to local tumor progression or distant failure), as well as pre-treatment prognostic and/or predictive factors.

\section{Sample size considerations}

The sample size is calculated with type I error of 0.025 , type II error of 0.2 (power of $80 \%$ ), and a one-sided hypothesis (only excess of cases is of interest). Conservatively assuming a 'normal' rate of $10 \%$ grade 3 or higher late toxicity, 70 patients are needed to exclude that the rate is actually $\geq 20 \%$, i.e., unacceptable, with the necessary precision. This sample size calculation is based directly on the binomial distribution. Adjusting the number of cases for ineligible or unanalyzable cases by $10 \%$, a minimum sample size of 77 patients is required for this study.

\section{Brachytherapy}

\section{Implantation procedure}

The implant procedure will be performed according to the standard technique. A Foley catheter will be inserted to visualize the urethra at the beginning of the procedure. The patient will be positioned in the lithotomy position, centered on the table. The ultrasound probe will be inserted into the rectum and adjusted to provide a satisfactory image without distorting the contour of the prostate. Row \#1 on the template matrix is positioned within the prostate on the axial image, on which the prostate is most posteriorly situated and is anterior to the rectum at all levels. Afterloading catheters may be placed with TRUS or MR guidance. The implant catheters must be non-me- tallic (for hyperthermia use). The implant procedure may be done under spinal or general anesthesia. During the implant, attention should be given to keep the catheters in the prostate without perforating the urethra. Posterior rows of catheters may be advanced into the seminal vesicles under TRUS guidance.

\section{Treatment planning transrectal ultrasonography}

Treatment planning will be based on a real time TRUS volume study of the prostate. It is acceptable also to use MRI based treatment planning. The ultrasound probe will be advanced until the base of the prostate is visualized, and this level will be designated the base plane. Contiguous serial axial images of the prostate at $\leq 3 \mathrm{~mm}$ increments beginning $5-10 \mathrm{~mm}$ cranial to the base plane (including all the catheters tips) will be obtained and will extend $>5 \mathrm{~mm}$ inferior (caudal) to the apex of the prostate. The CTV will be outlined on each axial image where it is present. The use of dwell time in dwell positions located outside of the PTV is discouraged to minimize normal tissue irradiation. A dwell time optimization program based on geometric or inverse and manual planning algorithm may be used.

The target volume definitions are largely based on those provided in ICRU Report 58: Dose and Volume Specification for reporting interstitial therapy [19]. The Clinical Target Volume (CTV) for stages T1-T2 includes the TRUS-defined prostate volume. For T3 stages, the CTV includes the whole prostate gland and extracapsular/seminal vesicle invasion. The Planning Target Volume (PTV) is the same as CTV. Additional 1-3 mm margins may be added in case of uncertainty of extracapsular infiltration.

\section{Dose specifications, dose fractionation}

In this trial, the dose prescribed to the PTV will be $30 \mathrm{~Gy}$ in three 10 Gy fractions spaced 21 days apart for HDRBT (the most common fractionation scheme in Poland) and 60 Gy in 2 fractions spaced 28 days apart for PDRBT (scheme from Erlangen). In both protocols, the brachytherapy will follow interstitial hyperthermia treatment.

To compare the expected biological effect of different fractionation schedules, the calculations were made using the linear-quadratic model [20] for the total biologically equivalent doses in 2-Gy fractions EQD2 both for HDRBT and PDRBT with multiple fractions (see Table 2).

\section{Dose-volume histogram (DVH) parameters, reporting}

The following dose parameters for CTV must be reported: $D_{90}, D_{M I N}, V_{100}, V_{150}, V_{200}$. The essential conditions are: $\mathrm{D}_{90}>100 \%, \mathrm{~V}_{100}>90-95 \%$. The required $\mathrm{DVH}$ parameters for the rectum and bladder (if contoured) are $\mathrm{D}_{0,1 \mathrm{cc}} \mathrm{D}_{1 \mathrm{cc}}, \mathrm{D}_{2 \mathrm{cc}}$. The dose constraint for the rectum is $\mathrm{D}_{2 \mathrm{cc}}$ $<50$ Gy $\left(E_{2}\right.$ for $\alpha / \beta=3$ ) (for HDRBT $D_{2 c c}<7.75$ Gy per fraction), and for the bladder is $\mathrm{D}_{2 \mathrm{cc}}<60$ Gy (EQD2 for $\alpha / \beta=3$ ) (for HDRBT $D_{2 c c}<8.6$ Gy per fraction). The urethra DVH parameters are $\mathrm{D}_{0,1 \mathrm{cc},} \mathrm{D}_{10}$, and $\mathrm{D}_{30}$. The dose constraint is $\mathrm{D}_{0,1 \mathrm{cc}}<120 \%$ of the prescribed dose. 
Table 2. Comparison of equivalent doses in 2-Gy fractions (EQD2) of the proposed treatment schemes

\begin{tabular}{lccc} 
& $\alpha / \beta=1$ Gy & $\alpha / \beta=3$ Gy & $\alpha / \beta=10$ Gy \\
\hline EQD2Gy (PDR: $2 \times 30 \mathrm{~Gy}=2 \times(50 \times 0.6 \mathrm{~Gy})) ; \mathrm{T}_{1 / 2}=1.9 \mathrm{~h}$ & 79.08 & 71.45 & 64.77 \\
\hline EQD2Gy (HDR: $30 \mathrm{~Gy}=3 \times 10 \mathrm{~Gy})$ & 110.00 & 78.00 & 50.00
\end{tabular}

$\alpha / \beta$-alpha/beta ratio, $T_{1 / 2}$ - half-time to repair, HDR - high-dose-rate brachytherapy, PDR - pulsed-dose-rate brachytherapy

\section{Hyperthermia}

Interstitial hyperthermia (IHT) treatment is scheduled before every brachytherapy fraction and it should end no more than one hour before the start of irradiation. Hyperthermia will be carried out in accordance with the QA guidelines published by the RTOG for interstitial hyperthermia [21]. Catheters implanted to the prostate during the implantation procedure will be used to place microwave antennas and temperature probes. At least eight, but as many catheters as possible should be placed in the prostate for microwave antennas and thermal sensors. The distance between antennas should be at least $5 \mathrm{~mm}$ and at most $15 \mathrm{~mm}$.

Thermometry probes should be positioned in the rectum and urethra near the prostate implant catheters, inside the urinary catheter, and in the prostate tissue (at least 2 probes) for continuous thermometry and thermal mapping of tumor-related temperatures. The objective of treatment is to achieve a tumor/prostate temperature of $40-47^{\circ} \mathrm{C}$. Therapeutic time starts when $40^{\circ} \mathrm{C}$ is achieved in at least one of the prostatic thermometry sensors or 30 minutes after enabling power. Planned treatment time is 60 minutes, and a maximum duration of 90 minutes of applied power is permitted. Failure to achieve $40^{\circ} \mathrm{C}$ in at least one prostate location for 30 minutes in at least two sessions will make the case not evaluable for response and toxicity. Hyperthermia withdrawal or shortening therapeutic time to 30 minutes is permitted in case of: 1 . early weakening of spinal anaesthesia; 2 . excessive patient mobility (risk of catheter displacement) or 3. patient request.

\section{Treatment schedules}

For HDR brachytherapy:

Day 1: IHT for 60 minutes followed by HDR within $60 \mathrm{~min}$; Day 22: IHT for 60 minutes followed by HDR within $60 \mathrm{~min}$; Day 43: IHT for 60 minutes followed by HDR within $60 \mathrm{~min}$.

For PDR brachytherapy:

Day 1-3: IHT 60 minutes followed by PDR within $60 \mathrm{~min}$; Day 29-31: IHT 60 minutes followed by PDR within $60 \mathrm{~min}$.

\section{Study duration}

The protocol was activated in February 2014 after receiving the vote of Ethics Committee of Chamber of Physicians in Krakow. The duration of patient recruiting is scheduled for approximately 3-4 years.

\section{Conclusions}

The standard dose prescription and schedule are not established yet for salvage brachytherapy re-irradiation of locally recurrent prostate cancer. The literature presents a wide range of treatment protocols. Consequently, a prospective trial is urgently needed to attain clear structured prospective data. The purpose of this report is to introduce a new prospective phase 2 trial combining Iridium-192 brachytherapy with interstitial hyperthermia (IHT). The primary aim of this trial is to analyze toxicity of the combined treatment, and the secondary aim is to define the efficacy (bNED, DFS, OS) of salvage brachytherapy and heat. The radiation dose prescribed to the PTV is 30 Gy in 3 fractions for HDR-brachytherapy and 60 Gy in 2 fractions for PDR-brachytherapy. The thermal dose prescribed to the PTV is a range of $40-47^{\circ} \mathrm{C}$ for $60 \mathrm{~min}$. The protocol anticipates treatment of 77 patients and the trial is currently open for participation by additional study centers.

\section{Disclosure}

Authors report no conflict of interest.

\section{References}

1. Kuban DA, el-Mahdi AM, Schellhammer PF. Prognosis in patients with local recurrence after definitive irradiation for prostatic carcinoma. Cancer 1989; 63: 2421-2425.

2. D'Amico AV, Whittington R, Malkowicz SB et al. Biochemical outcome after radical prostatectomy, eternal beam radiation therapy, or interstitial radiation therapy for clinically localized prostate cancer. JAMA 1988; 280: 969-974.

3. Lee WR, Hanks GE, Hanlon A. Increasing prostate specific antigen profile following definitive radiation therapy for localized prostate cancer: clinical observations. J Clin Oncol 1997; 15: 230-238.

4. Roach M, Hanks G, Thames H, et al. Defining biochemical failure following radiotherapy with or without hormonal therapy in men with clinically localized prostate cancer: recommendations of the RTOG-ASTRO Phoenix Consensus Conference. Int J Radiat Oncol Biol Phys 2006; 65: 965-974.

5. Arrayeh E, Westphalen AC, Kurhanewicz J et al. Does local recurrence of prostate cancer after radiation therapy occur at the site of primary tumor? Results of a longitudinal MRI and MRSI study. Int J Radiat Oncol Biol Phys 2012; 82: 787-793.

6. Ramey SJ, Marshall DT. Re-irradiation for salvage of prostate cancer failures after primary radiotherapy. World J Urol 2013; 31: 1339-1345.

7. Nguyen PL, D'Amico AV, Lee AK et al. Patient selection, cancer control, and complications after salvage local therapy for postradiation prostate-specific antigen failure: A systematic review of the literature. Cancer 2007; 110: 1417-1427.

8. Lukka H, Waldron T, Chin J et al. High-intensity focused ultrasound for prostate cancer: a practice guideline. Can Urol Assoc J 2010; 4: 232-236.

9. Yamada Y, Rogers L, Demanes DJ et al. American Brachytherapy Society consensus guidelines for high-dose-rate prostate brachytherapy. Brachytherapy 2012; 11: 20-32.

10. Łyczek J, Kawczyńska MM, Garmol M et al. HDR brachytherapy as a solution in recurrences of locally advanced prostate cancer. J Contemp Brachytherapy 2009; 1: 105-108. 
11. Scala L, Page PN, Lee JW. Salvage high dose rate brachytherapy for locally recurrent prostate cancer after primary radiotherapy. Int J Rad Onc Biol Phys 2009; 75: s322.

12. Jo Y, Fujii T, Hara R et al. Salvage high-dose-rate brachytherapy for local prostate cancer recurrence after radiotherapy - preliminary results. BJUI 2011; 109: 835-839.

13. Morikawa LK, Zelefsky MJ, Cohen GN et al. Salvage highdose-rate brachytherapy for recurrent prostate cancer after ultra high intensity modulated radiotherapy: results of a prospective study. Brachytherapy 2011; 10: S19.

14. Chen CP, Weinberg V, Shinohara $\mathrm{K}$ et al. Salvage HDR brachytherapy for recurrent prostate cancer after previous definitive radiation therapy: 5-year outcomes. Int J Radiat Oncol Biol Phys 2013; 86: 324-329.

15. Lahmer G, Lotter M, Kreppner S et al. Protocol-based image-guided salvage brachytherapy: Early results in patients with local failure of prostate cancer after radiation therapy. Strahlenther Onkol 2013; 189: 668-674.

16. Kukiełka AM, Hetnał M, Dąbrowski T et al. Salvage prostate HDR brachytherapy combined with interstitial hyperthermia for local recurrence after radiation therapy failure. Strahlenther Onkol 2013; 190: 165-170.

17. Ryu S, Brown SL, Kim SH et al. Preferential radiosensitization of human prostatic carcinoma cells by mild hyperthermia. Int J Radiat Oncol Biol Phys 1996; 34: 133-138.

18. Common Terminology Criteria for Adverse Events (CTCAE) Version 4.0; available at: http://ctep.cancer.gov/protocolDevelopment/electronic_applications/ctc.htm\#ctc_40

19. Dose and volume specification for reporting interstitial therapy in International Commission on Radiation Units and Measurements. ICRU Report 58, Bethesda 1997.

20. Joiner MC, Bentzen SM. Time-dose relationship: the linear quadratic approach. Arnold, London 2002.

21. Emami B, Stauffer P, Dewhirst MW et al. RTOG quality assurance guidelines for interstitial hyperthermia. Int J Radiat Oncol Biol Phys 1991; 20: 1117-1124. 